fachschaft.ufa@yandex.ru angefordert werden. Auch über Reaktionen und Fragen freue ich mich.

(C) Steppan, A., 2021 г.

НЕМЕЦКОЯЗЫЧНОЕ ОБРАЗОВАНИЕ И ДИАЛОГ КУЛЬТУР

УДК $81(07)$

DOI: 10.33184/NYVB-2021-04-06.12

Алина Т.Н.

(учитель нем.яз. МБОУ «Гимназия № 86», г. Уфа)

\title{
KREATIVES SCHREIBEN ALS DIE ART DES KREATIVEN DENKENS
}

B статье рассматриваются подходы $\kappa$ формированию креативного мышиения школьников как части мониторинга функциональной грамотности. Обосновывается возможность использования при этом технологии креативного письма. Дана краткая характеристика приемов технологии креативного письма.

Ключевые слова: Eine qualitativ hochwertige Bildung, vergleichende internationale Studien, PISA, kreatives Denken, die funktionale Grundbildung, Kreatives Schreiben, Schreibfähigkeiten, Kompetenzen, Impulse, Schreibanlässe.

Eine qualitativ hochwertige Bildung ist die Aufgabe, mit der die russische Schulbildung konfrontiert war und ist. Der Text des Dekrets des russischen Präsidenten Wladimir Putin von 2018 "Über nationale Ziele und strategische Ziele der Entwicklung der Russischen Föderation für den Zeitraum bis 2024" besagt, dass die Regierung der Russischen Föderation bei der Entwicklung des nationalen Projekts im Bildungsbereich davon ausgehen muss, dass im Jahr 2024 die Erreichung die folgenden Ziele und Vorgaben sichergestellt werden muss: die Gewährleistung der globalen Wettbewerbsfähigkeit der 
russischen Bildung, der Beitritt der Russischen Föderation zu den zehn Ländern der Welt in Bezug auf die Qualität der allgemeinen Bildung.

Wie wird die Qualität der Bildung in Russland bewertet? Welche Methoden werden dafür verwendet? Die wichtigsten Indikatoren, anhand derer die Position Russlands im Verhältnis zu anderen Ländern der Welt in Bezug auf die Qualität der allgemeinen Bildung bestimmt und die Wettbewerbsvorteile russischer Schulkinder aufgezeigt werden, sind vergleichende internationale Studien zur Qualität von Allgemeinbildung. Die häufigsten Studien sind PIRLS (Internationale Studie zum Leseverständnis), TIMSS (Internationale Studie, wo mathematische und naturwissenschaftliche Fähigkeiten der Schülerinnen und Schüler untersucht werden) und natürlich PISA (Internationales Programm, Forschungsprogramm zum internationalen Vergleich von Schülerleistungen am Ende der obligatorischen Schulzeit).

Die Schulkinder aus Baschkortostan werden ebenfalls bewertet, die Studie wird im Jahr 2024 stattfinden. Das Verfahren zur Bewertung der Qualität der Ausbildung von Studenten in Baschkortostan wird unter Verwendung der regionalen Bewertung gemäß dem PISA-Modell durchgeführt, d. h. unter Berücksichtigung der Praxis internationaler vergleichender Studien zur Bildungsqualität, vor allem aber auf der Grundlage des staatlichen Bildungsstandards und der Massenbewertungsverfahren in der Russischen Föderation.

PISA ist das größte internationale Programm zur Bewertung von Bildungsleistungen (Programme for International Student Assessment), das unter der Schirmherrschaft der Organisation für wirtschaftliche Zusammenarbeit und Entwicklung (OECD) durchgeführt wird. Die Studie prüft mathematische und naturwissenschaftliche Kenntnisse, die Lesekompetenz und Finanzkenntnisse. 


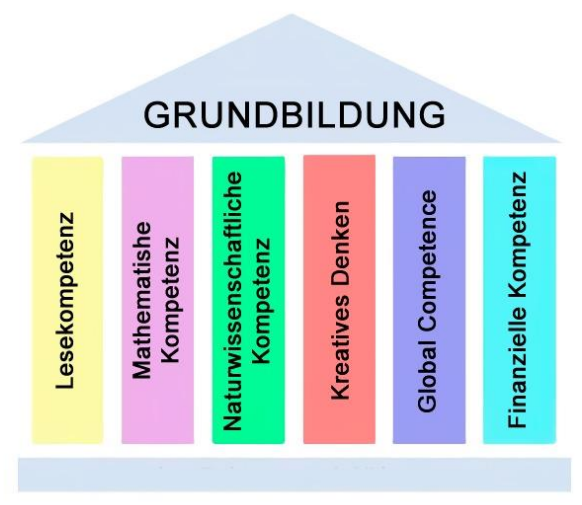

Im Jahr 2021 wird erstmals die Bewertung des kreativen Denkens als eine der Hauptkomponenten in die PISA-Studie aufgenommen. Warum wird es empfohlen, kreatives Denken zu evaluieren? Was ist die Motivation für diese Entscheidung?

Alle diese Forschungsthemen, die auf die Beurteilung der Qualität der Bildung abzielen, sind Bestandteile der modernen funktionalen Grundbildung, der sehr gut widerspiegelt, wie viel moderne Lernende wissen und können sollten. Das Konzept der funktionalen Grundbildung wurde erstmals 1965 auf dem Weltkongress der Bildungsminister in Teheran angewendet, und dann ging es um eine Reihe von Fähigkeiten zum Lesen und Schreiben zur Verwendung im Alltag und zur Lösung alläglicher Probleme. 1978 ergänzt die UNESCO dieses Konzept: Nur einer gilt als funktional gebildet, der an allen Aktivitäten teilnehmen kann, bei denen Alphabetisierung für das effektive Funktionieren seiner Gruppe erforderlich ist. Dies ermöglicht ihm auch, weiterhin Lesen, Schreiben und Zählen sowohl für eigene Entwicklung, sondern auch für die weitere Entwicklung seiner Gruppe, seines sozialen Umfeldes zu verwenden.

Man versteht daher, dass der Staat eine Forderung nach der funktionalen Grundbildung der Schülerinnen und Schüler hat, weil die funktionale Grundbildung die Fähigkeit ist, das in der Schule erworbene Wissen anzuwenden, um alltägliche Probleme zu lösen. Die Einbeziehung der Bewertung des kreativen Denkens in die Studie der funktionalen Grundbildung ermöglicht positive Änderungen in der Praxis der Lehr- und Bildungspolitik, die in Zukunft zur Lösung der 
strategischen Aufgaben der sozioökonomischen Entwicklung des Landes beitragen werden.

Kreatives Denken ist eine Art des Denkens, das mit dem Schaffen oder Entdecken von etwas Neuem verbunden ist, das es ermöglicht, die Welt auf eine andere Art und Weise zu betrachten, neue Ansätze zur Lösung von Problemen zu finden und erfolgreich mit den Herausforderungen der Welt umzugehen. Die Fähigkeit zu kreativem Denken ist die Grundlage für die Entwicklung aller Bereiche der menschlichen Kultur, d. h. alles hängt von der Entstehung innovativer Ideen $a b$, von der Schaffung neuen Wissens und neuer Technologien.

Jeder Mensch besitzt die Fähigkeit, mehr oder weniger kreativ zu denken. Die Gewohnheit, kreativ zu denken, korreliert mit der Beteiligung an produktiven Aktivitäten, leistet einen unschätzbaren Beitrag zur Entwicklung aller Aspekte der Persönlichkeit. Die Gewohnheit, kreativ zu denken, hilft den Menschen, bessere Ergebnisse bei der Transformation der umgebenden Realität $\mathrm{zu}$ erzielen. Es ist wichtig, dass die Fähigkeit zum kreativen Denken auf Wissen und Erfahrung beruht und daher der Gegenstand einer gezielten Ausbildung sein kann.

Dies bedeutet, dass die Lehrkräfte der Fremdsprache sich in ihrem Unterricht mit der Entwicklung des kreativen Denkens bei den Lernenden beschäftigen sollten, für diese Art des Denkens, wenn sich eine Person darauf konzentrieren kann, Ideen $\mathrm{zu}$ erforschen und verschiedene Möglichkeiten zu generieren. Die Lehrkräfte können ihren SuS helfen, zu träumen und sich der Erfüllung von Träumen zu nähern, die Träume zu bewerten und zu verbessern, bis sie wirklich werden.

In der modernen Methode des Fremdsprachenunterrichts, insbesondere des Schreibens in einer Fremdsprache, verbreitet sich die Technologie des kreativen Schreibens - eine Art des Schreibens, die über die Grenzen der akademischen oder technischen Literatur hinausgeht und durch die Erzählform, durch die Schaffung und Entwicklung von Charakteren, Verwendung verschiedener Ausdrucksmittel usw. bestimmt wird. Bei der Bildung des kreativen Schreibens spielen produktive Übungen unterschiedlicher Komplexität eine wichtige Rolle, 
die sich in Form und Inhalt unterscheiden und oft eine spielerische Form haben. Natürlich werden diese Übungen nur schriftlich gemacht, inhaltlich sind sie sprach- oder halbkreativ.

Wenn man das Schreiben im Allgemeinen als eine produktive Kommunikationsaktivität betrachtet, die dank den persönlichen Motiven (z. B. einen Brief schreiben) oder durch ein soziales Bedürfnis (einen Bericht schreiben) realisiert wird, dann scheinen geschriebene Übungen mit der kommunikativen Orientierung im Fremdsprachenunterricht logischer. Der Aktivitätsansatz ermöglicht es dann, den Prozess des Beherrschens einer fremdsprachigen schriftlichen Rede gemäß den Prinzipien des persönlichen Aktivitätslernens zu organisieren: Konzentration auf den Prozess und nicht auf das Ergebnis der Aktivität, die Unabhängigkeit der SuS bei der Auswahl des Inhalts und der sprachlichen Form seiner eigenen kreativen schriftlichen Arbeiten (Essays, Geschichten, Märchen, Gedichte usw.). Mit einem aktivitätsbasierten Ansatz für das Unterrichten von der schriftlichen Fremdsprache besteht die Aufgabe der Lehrkraft darin, die SuS zu kreativer Arbeit zu motivieren und sie mit verschiedenen Strategien und Arbeitstechniken vertraut zu machen.

Kreatives Schreiben ermöglicht es den Lernenden, vorhandene lexikalische und grammatikalische Fähigkeiten zu nutzen, ihre Individualität auszudrücken, sich interkultureller Unterschiede bewusst $\mathrm{zu}$ werden und ihre eigenen schriftlichen Arbeiten zu verwenden, um ihre Lese- und Sprechfähigkeiten zu trainieren. Der Zweck dieses Prozesses ist es, einen Text $\mathrm{zu}$ erstellen, der auf der individuellen Kreativität der SuS basiert. Beispiele für kreatives Schreiben:

- Schreibe einen Monolog für eine Socke, die versehentlich in einem Kühlschrank voller Lebensmittel den Tag verbracht hat.

- Denke an den langweiligsten Tag deines Lebens. Beschreibe ihn, aber denke daran, dass deine Geschichte nicht so langweilig wie dein Tag sein sollte.

- Fange so an: «Wenn ich etwas ändern könnte, würde ich .... ändern».

- Beschreibe mit den Worten, die für heute gelernt wurden, das Aussehen, den Beruf und die Gewohnheiten der Person, der Chef Peter heißt. 
- Wohin gehst du, wenn du dich ausruhen willst? Schreibe über diesen Ort.

- Beschreibe den idealen Ort, wo du leben möchtest.

- Warum wechselt der Lehrer seinen Beruf? Nenne mindestens 10 Gründe.

Wenn man kreativ wird, kann man nicht nur Informationen präsentieren. Man verwendet dabei die Vorstellungskraft, drückt Emotionen aus, um originelle Dinge zu schaffen oder bestehende Ideen auf neue Weise zu nutzen. Es ist viel mehr als nur Poesie und Prosa. Die Hauptsache ist, dass das kreative Schreiben für jeden geeignet ist, da es in seinen Genres, seiner Größe und seinem Schwierigkeitsgrad unglaublich vielfältig ist. Und alle Menschen sind kreativ.

Das kreative Schreiben erweitert den Umfang des funktionalen Schreibens. Man schreibt kreativ, wenn man beschreibt, wie die Welt verstanden und gesehen wird. Auf diese Weise ist dies eine Technik, die erlebten Emotionen für die Zukunft zu bewahren. Weil kreatives Schreiben sich so sehr von anderen Arten des Schreibens unterscheidet, verbessert es das Verständnis der Sprache und zwingt, sie auf neue Art und Weise zu verwenden.

Dies gilt auch für das Erlernen einer Fremdsprache. Das Schreiben von Geschichten, ob real oder imaginär, ist eine großartige Möglichkeit, die neue Sprache zu üben. Der Wortschatz wird aktiv genutzt und erweitert. Wenn man beispielsweise Geschäftsbriefe schreibt, arbeitet man normalerweise mit Klischees.

Im Gegensatz zu akademischen Methoden des Schreibunterrichts, bei denen die SuS lernen, sich auf bestehende Sprachregeln zu verlassen, konzentriert sich kreatives Schreiben auf eine Idee. Beispielsweise sind folgende Aufgaben interessant:

\begin{tabular}{|l|l|l|}
\hline 1. Texte fortsetzen: & $\begin{array}{l}\text { 2. Texte } \\
\text { umschreiben, } \\
\text { verändern: }\end{array}$ & $\begin{array}{l}\text { 3. Paralleltexte, } \\
\text { Gegentexte } \\
\text { schreiben: }\end{array}$ \\
\hline $\begin{array}{l}\text { - fehlende Teile } \\
\text { ergänzen }\end{array}$ & - Textsorte ändern & $\begin{array}{l}\text { - vom Inhalt } \\
\text { ausgehend }\end{array}$ \\
\hline
\end{tabular}




\begin{tabular}{|l|l|l|}
\hline - Teile ändern & - Stilart ändern & $\begin{array}{l}\text { - von der äußeren } \\
\text { Form ausgehend }\end{array}$ \\
\hline $\begin{array}{l}\text { - Vorgeschichte, } \\
\text { Folgen ergänzen }\end{array}$ & - Adressat ändern & \\
\hline & - Personen einführen & \\
\hline & - Perspektive ändern & \\
\hline
\end{tabular}

Eine der gebräuchlichsten Methoden des kreativen Schreibens, wenn sich die Schreibfähigkeiten der SuS sowie ihr kreatives Potenzial entwickeln, ist die Gedichte zu schreiben, und zwar Kurzgedichte, z.B.: 1. Haiku bezeichnet ein extrem kurzes japanisches Gedicht. Diese Gedichtform gehört zum Kreativen Schreiben, weil man Haiku nutzt, um die Weltwahrnehmung auszudrücken.

2. Die Bildergedichte sind Gedichte, wo die Wörter teilweise mit den Bildern ersetzt sind.

3. Ein Elfchen ist ein kurzes Gedicht mit einer vorgegebenen Form: elf Wörter, die in festgelegter Folge auf fünf Zeilen verteilt werden. Man formuliert für jede Zeile eine Anweisung.

4. Ein Akrostichon - ist ein Gedicht, in dem die Anfangsbuchstaben des ersten Wortes in jedem Vers untereinander gelesen einen Sinn ergeben (ein Wort oder ein kurzer Satz, der thematisch mit dem ganzen Gedicht zusammenhängt).

Kreatives Schreiben kann in jeder Phase des Unterrichts eingesetzt werden. Dabei können verschiedene Arten von Unterstützungen empfohlen werden: visuelle Impulse (Gegenstände, Spielzeug, Bilder, Fotos, Zeichnungen, Bildergeschichten und Comics), akustische Impulse (Audiotexte, Lieder, instrumentale Musikstiucke usw.), geschriebene Impulse (Gedichte, Zitate, Sprichwörter, Redewendungen usw.). «Ein Bild sagt mehr als tausend Worte» ist ein Sprichwort und eine Metapher für den Mehrwert von Bildern gegenüber ausschließlichem Text.

Wen siehst du, eine junge Frau oder ein Alte? 

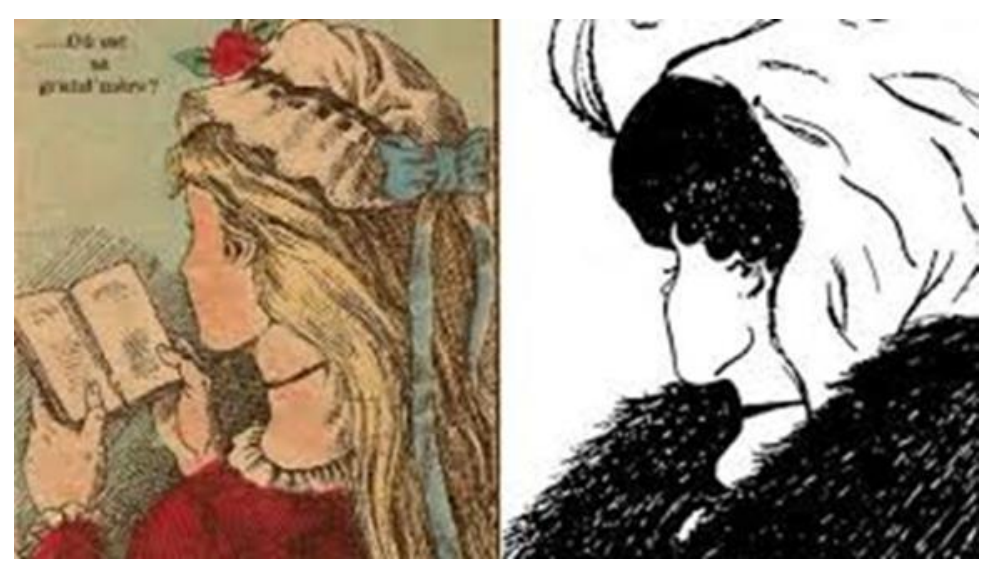

Wenn die SuS schon den guten Umgang mit dem kreativen Schreiben haben, kann man ohne Schreibanlässe, d.h. ohne Unterstützung kreativ schreiben. Zum Beispiel können SuS einen spontanen kreativen Beitrag darüber schreiben, was sie am meisten beunruhigt, amüsiert, überrascht, empört usw.

«Kreatives Schreiben» bedeutet kreativ mit der Sprache umzugehen. Die wichtigsten 10 Merkmale des kreativen Schreibens sind zu betrachten:

1. Kreatives Schreiben ist das Gegengewicht gegenüber einem normalen traditionellen Schreibunterricht.

2. Kreatives Schreiben ist für alle. Die kleinen Kinder mit dem geringen Wortschatz können schon kleine, aber aussagekräftige Texte schreiben.

3. Man kann eigene Identität kennen lernen, man öffnet das subjektive Konzept.

4. Kreatives Schreiben setzt Phantasie frei.

5. Kreatives Schreiben ist für alle. Man schreibt ohne Angst, weil man spielerisch mit der Sprache umgeht.

6. Kreatives Schreiben kann man erlernen, weil jeder Mensch ein kreatives Potenzial besitzt.

7. Kreatives Schreiben verbindet, macht Spaß.

8. Kreatives Schreiben knüpft an die Lebenswelt an.

9. Kreatives Schreiben fördert die Lesekompetenz, Erzählkompetenz.

10. Kreatives Schreiben hilft dabei, sich eine eigene Meinung zu bilden. 
Die sprachliche Kreativität kann durch Fördern und Üben unterstützt und eingeübt werden. Die Aufgabenstellung, einen Text zu verfassen, stellt SuS vor eine umfangreiche interessante Aufgabe.

Die Leistungstests der PISA-Studie basieren also auf dem Begriff der funktionalen Grundbildung. Im Kontext von PISA umfasst „funktional“ im Wesentlichen zwei Aspekte, nämlich die Anwendbarkeit für die jetzige und die spätere, nachschulische Teilhabe an einer Kultur sowie die Anschlussfähigkeit im Sinne kontinuierlichen Weiterlernens über die Lebensspanne. Kreatives Denken wird dabei als die Kompetenz definiert und hilft vielfältige, kreative Ideen $\mathrm{zu}$ produzieren, sie $\mathrm{zu}$ evaluieren und $\mathrm{zu}$ verbessern. Die Ideen können effektive Lösungen realer Probleme, Fortschritte im Wissen und wirkungsvolle Ausdrucksformen der Vorstellungskraft sein. In jedem Fall müssen diese aber neuartig und zweckmäßig sein. Und in diesem Artikel dargestellten Methoden und Techniken des kreativen Schreibens helfen bestimmt den Lernenden aus Baschkortostan, 2024 gute Ergebnisse der PISA-Studie zu bekommen.

\section{ЛИТЕРАТУРА}

1. Методология и критерии оценки качества общего образования в общеобразовательных организациях на основе практики международных исследований качества подготовки обучающихся (утв. приказами Рособрнадзора № 590, Минпросвещения России № 219 от 06.05.2019) (ред. от 24.12.2019) [Электронный ресурс]. URL:http://www.consultant.ru/document/cons_doc_LAW_325095 / (дата обращения: 11.04.2021).

2. Фролова П.И. К вопросу об историческом развитии понятия «Функциональная грамотность» в педагогической теории и практике// Наука о человеке: гуманитарные исследования. 2016. № 1 (23). [Электронный pecypc]. URL: https://cyberleninka.ru/article/n/k-voprosu-ob-istoricheskom- 
razvitii-ponyatiya-funktsionalnaya-gramotnost-v-

pedagogicheskoy-teorii-i-praktike (дата обращения: 11.04.2021).

3. Brenner, G. Texte schreiben: Alles klar! - Berlin: Cornelsen, 2004. - S. 80.

4. Gerngroß, G., Krenn W., Puchta H-Grammatik kreativ- Materialien für einen lernerzentrierten Grammatikunterricht. - Berlin: Langenscheidt, 1999. - S. 160.

5. Wolfrum, J. Kreativ Schreiben. Gezielte Schreibförderung für jugendliche und erwachsene Deutschlernende (DaF/DaZ). Ismaning: Hueber, 2010.

6. URL: https://www.pisa.tum.de/kompetenzbereiche/ (дата обращения: 11.04.2021).

(C) Т.Н. Алина, 2021 г.

УДК $81(07)$

DOI: 10.33184/NYVB-2021-04-06.13

Денисова Л.А. (ст. преп. БашГУ, г. Уфа)

\section{УРОК ЛАТИНСКОГО ЯЗЫКА У ИЗУЧАЮЩИХ НЕМЕЦКИЙ ЯЗЫК}

B cтатье рассматриваются основные дидактические приниипь и методические приемы обучения латинскому языку студентов факультета романо-германской филологии, изучающих западноевропейские иностранные языки (английский, немеикий, франиузский). Подчеркивается особая роль дисциплинь «Латинский язык» в освоении базовых филологических компетенций. Автор предлагает общий алгоритм изучения латинского языка на языковом факультете, а также даёт детальный план проведения лексико-грамматического анализа 